\title{
Minimally invasive versus open distal pancreatectomy for ductal adenocarcinoma (DIPLOMA): a pan-European propensity score matched study
}

J. van Hilst, MD, MSc ${ }^{1}$, T. de Rooij, $\mathrm{MSc}^{1}$, S. Klompmaker, $\mathrm{MD}^{1}$, M. Rawashdeh, MD², F. Aleotti, $\mathrm{MD}^{3}$, B. Al-Sarireh, MD, $\mathrm{PhD}^{4}$, A. Alseidi, $\mathrm{MD}^{5}$, Z. Ateeb, $\mathrm{MD}^{6}$, G. Balzano, $\mathrm{MD}^{3}$, F. Berrevoet, MD, $\mathrm{PhD}^{7}$, B. Björnsson, $\mathrm{MD}^{8}$, U. Boggi, $\mathrm{MD}^{9}$, O.R. Busch, $\mathrm{MD}, \mathrm{PhD}^{1}, \mathrm{G}$. Butturini, $\mathrm{MD}, \mathrm{PhD}^{10}$, R. Casadei, $\mathrm{MD}, \mathrm{PhD}^{11}$, M. del Chiaro, MD, $\mathrm{PhD}^{6}$, F. Cipriani, $\mathrm{MD}^{2}$, R. van Dam, MD, $\mathrm{PhD}^{12}$, I. Damoli, $\mathrm{MD}^{13}$, S. van Dieren, $\mathrm{PhD}^{1}$, S. Dokmak, $\mathrm{MD}^{14}$, B. Edwin, $\mathrm{MD}, \mathrm{PhD}{ }^{15}$, C. van Eijck, MD, PhD ${ }^{16}$, J.M. Fabre, MD, $\mathrm{PhD}^{17}, \mathrm{M}$. Falconi, $M D, \mathrm{PhD}^{3}$, O. Farges, $\mathrm{MD}, \mathrm{PhD}^{14}$, L. Fernández-Cruz, $\mathrm{MD}^{18}, \mathrm{~A}$. Forgione, $\mathrm{MD}, \mathrm{PhD}^{19}$, I. Frigerio, $M D^{10}$, D. Fuks, MD, PhD ${ }^{20}$, F. Gavazzi, $\mathrm{MD}^{21}$, B. Gayet, $\mathrm{MD}, \mathrm{PhD}^{20}, \mathrm{~A}$. Giardino, $\mathrm{MD}^{10}, \mathrm{~B}$. Groot Koerkamp, MD, $\mathrm{PhD}^{16}$, T. Hackert, $\mathrm{MD}^{22}$, M. Hassenpflug, $\mathrm{MD}^{22}$, I. Kabir, $\mathrm{MD}^{23}$, T. Keck, $\mathrm{MD}^{24}$, I. Khatkov, MD, PhD ${ }^{25}$, A. Klock, $\mathrm{PhD}^{26}, \mathrm{M}$. Kusar, $\mathrm{MD}^{27}, \mathrm{C}$. Lombardo, $\mathrm{MD}^{9}$, G. Marchegiani, $\mathrm{MD}^{13}, \mathrm{R}$. Marshall, $\mathrm{MD}^{5}$, K.V. Menon, MD, $\mathrm{PhD}^{28}$, M. Montorsi, $\mathrm{MD}^{21}$, M. Orville, $\mathrm{MD}^{14}$, A. Pietrabissa, $\mathrm{MD}^{29}$, I. Poves, MD, PhD ${ }^{30}$, J. Primrose, MD, PhD ${ }^{2}$, R. Pugliese, $\mathrm{MD}^{19}$, C. Ricci, $\mathrm{MD}^{11}$, K. Roberts, MD, PhD ${ }^{31}, \mathrm{~B}$. Røsok, MD, PhD ${ }^{15}, M A$. Sahakyan, $M D, P h D^{15}$, S. Sánchez-Cabús, $M D, P h D^{18}, P$. Sandström, MD, $P h D^{8}$, L. Scovel, $M D^{5}$, L. Solaini, $M D^{32}$, Z. Soonawalla, $M D^{23}$, F.R. Souche, $M D^{17}$, R. Sutcliffe, $M D^{31}$, G. Tiberio, $\mathrm{MD}^{32}$, A. Tomazic, MD, $\mathrm{PhD}^{27}$, R. Troisi, MD, $\mathrm{PhD}^{7}, \mathrm{U}$. Wellner, $\mathrm{MD}^{24}$, S. White, $\mathrm{MD}^{33}$, U.A. Wittel, $M D^{26}$, A. Zerbi, $M D^{21}$, C. Bassi, $M D^{13}$, M.G. Besselink, MD, MSc, $\mathrm{PhD}^{1^{*}}, \mathrm{M}$. Abu Hilal, MD, $\mathrm{PhD}^{2^{*}}$ for the European Consortium on Minimally Invasive Pancreatic Surgery (E-MIPS)

* Principal investigators and shared senior authors

1. Department of surgery, Academic Medical Center, Amsterdam, the Netherlands

2. Department of surgery, Southampton University Hospital NHS Foundation Trust, Southampton, United Kingdom

3. Department of surgery, San Raffaele Hospital, Milan, Italy

4. Department of surgery, Morriston Hospital, Swansea, United Kingdom

5. Department of surgery, Virginia Mason Medical Center, Seattle, United States

6. Department of surgery, Karolinska Institute, Stockholm, Sweden

7. Department of surgery, Ghent University Hospital, Ghent, Belgium

8. Department of surgery, Linköping University, Linköping, Sweden 
9. Department of surgery, Universitá di Pisa, Pisa, Italy

10. Department of surgery, Pederzoli Hospital, Peschiera, Italy

11. Department of surgery, S. Orsola-Malpighi Hospital, Bologna, Italy

12. Department of surgery, Maastricht University Medical Center, Maastricht, the Netherlands

13. Department of surgery, Verona University Hospital, Verona, Italy

14. Department of surgery, Hospital of Beaujon, Clichy, France

15. Department of surgery, Oslo University Hospital and Institute for Clinical Medicine, Oslo, Norway

16. Department of surgery, Erasmus MC, Rotterdam, the Netherlands

17. Department of surgery, Hopital Saint Eloi, Montpellier, France

18. Department of surgery, Hospital Clínic de Barcelona, Barcelona, Spain

19. Department of surgery, Niguarda Ca' Granda Hospital, Milan, Italy

20. Department of surgery, Institut Mutualiste Montsouris, Paris, France

21. Department of surgery, Humanitas University Hospital, Milan, Italy

22. Department of surgery, Heidelberg University Hospital, Heidelberg, Germany

23. Department of surgery, Oxford University Hospital NHS Foundation Trust, Oxford, United Kingdom

24. Department of surgery, Lübeck University Hospital, Lübeck, Germany

25. Department of surgery, Moscow Clinical Scientific Center, Moscow, Russian Federation

26. Department of surgery, Universitätsklinikum Freiburg, Freiburg, Germany

27. Department of surgery, University Medical Center Ljubljana, Ljubljana, Slovenia

28. Department of surgery, King's College Hospital NHS Foundation Trust, London, United Kingdom

29. Department of surgery, University hospital Pavia, Pavia, Italy

30. Department of surgery, Hospital del Mar, Barcelona, Spain

31. Department of surgery, University Hospital Birmingham, Birmingham, United Kingdom

32. Department of surgery, University of Brescia, Brescia, Italy

33. Department of surgery, The Freeman Hospital Newcastle Upon Tyne, Newcastle, United Kingdom

\section{Corresponding author:}

Mohammed Abu Hilal, MD PhD FRCS FACS Southampton University Hospital NHS

Foundation Trust

Tremona Road, SO16 2YD

Southampton, UK

Tel: +44-23-8077-7222

Email: abuhilal9@gmail.com
During review process:

Jony van Hilst, MD, MSc

Academic Medical Center

Department of surgery, G4-136

PO BOX 22660

1100 DD Amsterdam

The Netherlands

$+3125661388$ 
Marc G Besselink, MD, MSc, PhD

j.vanhilst@amc.nl

Academic Medical Center

Department of surgery, G4-196

PO BOX 22660

1100 DD Amsterdam

The Netherlands

$+3125662558$

m.g.besselink@amc.nl

Running head (39/40): Minimally invasive vs. open DP for PDAC

Conflicts of Interest: None

Funding source: No specific funding obtained

\section{Mini-abstract (42/50)}

Data on oncological safety of minimally invasive distal pancreatectomy (MIDP) for pancreatic ductal adenocarcinoma (PDAC) are scarce. This pan-European propensity score matched study found higher R0-resection rates, lower lymph node retrieval and comparable survival after MIDP vs. open distal pancreatectomy for PDAC. 


\section{Structured Abstract (250/250 words)}

Objective: To compare oncological outcomes after minimally invasive distal pancreatectomy (MIDP) vs. open distal pancreatectomy (ODP) in patients with pancreatic ductal adenocarcinoma (PDAC).

Summery Background Data: Cohort studies have suggested superior short-term outcomes of MIDP compared with ODP. Recent international surveys, however, revealed that surgeons have concerns about the oncological safety of MIDP for PDAC.

Methods: A pan-European propensity score matched (PSM) study including patients who underwent MIDP or ODP for PDAC between January $1^{\text {st }}, 2007$ and July $1^{\text {st }}, 2015$. MIDP patients were matched to ODP patients in a 1:1 ratio. Main outcomes were radical (R0) resection, lymph node retrieval, and survival.

Results: In total, 1212 patients were included from 34 centers in 11 countries. Out of 356(29\%) MIDP patients, 340 could be matched to an ODP control. After matching, the MIDP conversion rate was $19 \%(\mathrm{n}=62)$. Median blood loss (200mL[60-400] vs. $300 \mathrm{~mL}[150-500], P=0.001)$ and hospital stay (8[6$12]$ vs. $9[7-14]$ days, $P<0.001)$ were less after MIDP. Clavien-Dindo grade $\geq 3$ complications ( $18 \%$ vs. $21 \%, P=0.431)$ and 90 -day mortality ( $2 \%$ vs. $3 \%, P>0.99)$ were comparable for MIDP and ODP respectively. R0 resection rate was higher ( $67 \%$ vs. $58 \%, P=0.019$ ), whereas Gerota's fascia resection rate $(31 \%$ vs. $60 \%, P<0.001)$ and lymph node retrieval $(14[8-22]$ vs. $22[14-31], P<0.001)$ were lower after MIDP. Median overall survival was 28 vs. 31 months ( $P=0.929)$.

Conclusion: Although survival did not differ between MIDP and ODP for PDAC, the opposing differences in RO resection rate, resection of Gerota's fascia and lymph node retrieval require confirmation of the oncological safety of MIDP in a randomized trial. 


\section{Introduction}

Minimally invasive distal pancreatectomy (MIDP), defined as either laparoscopic or robot-assisted distal pancreatectomy, was introduced in $1994 .{ }^{1}$ Several systematic reviews of cohort studies have suggested superior short-term outcomes of MIDP as compared to open distal pancreatectomy (ODP) for non-malignant pancreatic diseases, without increasing costs. ${ }^{2-11}$ The most important advantages of MIDP include less intraoperative blood loss and shorter postoperative hospital stay. However, the oncological safety in terms of resection margins, adequate lymphadenectomy, and survival after MIDP in the treatment of pancreatic ductal adenocarcinoma (PDAC) remains controversial.

A recent Cochrane review including 11 studies and a total of 1506 patients with PDAC of the pancreatic body or tail showed comparable rates of non-radical (R1/R2) resection margins, tumor recurrence, and survival after MIDP and ODP. ${ }^{12}$ Importantly, as randomized controlled trials were lacking most studies were single-center and retrospective, leading to concerns on the impact of treatment allocation bias. Further concerns on the oncological outcomes of MIDP for patients with PDAC were raised in two recent international surveys. ${ }^{13,14}$ Almost one third of European pancreatic surgeons considered MIDP inferior to ODP regarding oncological outcomes ${ }^{13}$ and a worldwide survey showed that $21 \%$ of pancreatic surgeons considered PDAC a contra-indication for a minimally invasive aproach ${ }^{14}$. Surgeons may doubt whether the essential components of an adequate oncological resection during distal pancreatectomy (i.e. radical resection margins, resection of Gerota's fascia, splenectomy and sufficient lymphadenectomy) are equally well obtained during MIDP compared to ODP.

In 2015, a group of European surgeons initiated the European Consortium for Minimally Invasive Pancreatic Surgery (E-MIPS) in order to safely implement minimally invasive pancreatic surgery. This group designed the DIPLOMA project (Distal Pancreatectomy, minimally invasive or open for 
malignancy), which aims to compare short and long term outcomes after MIDP and ODP in patients with PDAC with a focus on resection margins, lymphadenectomy and survival. 


\section{Methods}

This study was performed according to the Strengthening the Reporting of Observational Studies in Epidemiology (STROBE) guidelines. ${ }^{15}$ The ethics committee of the Academic Medical Center waived the need for informed consent due to the observational study design.

\section{$\underline{\text { Design and patients }}$}

This pan-European retrospective cohort study was performed within centers of the E-MIPS. All consecutive patients who underwent distal pancreatectomy (minimally invasive or open) with a histopathological diagnosis of PDAC between January $1^{\text {st }}, 2007$ and July $1^{\text {st }}, 2015$ were eligible for inclusion. Patients were excluded if they had a previous pancreatic resection, if distant metastasis were present, if the tumor involved the celiac trunc or when the tumor only became resectable after down staging with neo-adjuvant therapy. Patients were categorized according to the method of surgery: MIDP or ODP.

\section{Definitions}

MIDP was defined as laparoscopic or robot-assisted surgery. PDAC was defined according to the WHO classification of pancreatic tumors ${ }^{16}$. MIDP conversion was defined as any laparotomy for other reasons than trocar placement or specimen extraction. Postoperative complications were classified using the Clavien-Dindo classification. ${ }^{17}$ Major complications were defined as Clavien-Dindo grade 3 or higher. The definitions for pancreatic surgery specific complications of the International Study Group on Pancreatic Surgery (ISGPS) were used to score postoperative pancreatic fistula (POPF), delayed gastric emptying (DGE) and postpancreatectomy hemorrhage (PPH). ${ }^{18-20}$ Only ISGPS grade B/C complications were considered clinically relevant and subsequently registered. Surgical site infection (SSI) was defined using the Centers for Disease Control and Prevention (CDC) definition. ${ }^{21}$ Resection margins, including transection and circumferential margins, were categorized into; RO (distance margin to tumor $\geq 1 \mathrm{~mm}$ ), R1 (distance margin to tumor $<1 \mathrm{~mm}$ ) and R2 (macroscopically positive margin) according to the Royal College of Pathologists definition. ${ }^{22}$ 


\section{Data collection}

All 34 participating centers received a blank database including all parameters of interest. The data were collected locally by each participating center and combined centrally by the study coordinators. The participating centers received a survey regarding the method of local data collection (e.g. type of database used and annual volumes). Baseline characteristics collected included sex, age, body mass index $\left(\mathrm{BMI}, \mathrm{kg} / \mathrm{m}^{2}\right)$, previous abdominal surgery, American Society of Anaesthesiologists (ASA) physical status, tumor location, tumor size $(\mathrm{mm})$ and tumor involvement of other organs on preoperative imaging and administration of neoadjuvant therapy. Collected outcomes were procedure type (open, minimally invasive), conversion and reason for conversion, operative time (min.), blood loss $(\mathrm{mL})$, splenectomy, resection of Gerota's fascia, adrenalectomy, additional organ resection (beyond adrenalectomy and splenectomy), vascular resection (beyond resection of the splenic vessels), tumor size ( $\mathrm{mm})$, overall and tumor positive lymph node retrieval, tumor and lymph node stage, involvement of resection margin, lymphovascular and perineural invasion, major complications, POPF, DGE, PPH, SSI, length of hospital stay (days), readmission, 90-day mortality, adjuvant chemotherapy, time until start adjuvant chemotherapy (days) and overall survival (months). Complications, re-admissions and mortality were all collected up to 90-days postoperatively. Overall survival was, depending on the center, either collected from patient records, municipal personal records database, or by personal contact with patient or family. All data were stored and processed anonymously.

\section{Matching}

To minimize the impact of treatment allocation bias, MIDP patients were matched to ODP patients using propensity scores. Multivariable logistic regression was performed to estimate the propensity scores for the intervention group (MIDP as primary treatment). Baseline variables available (age, sex, BMI, ASA physical status, prior abdominal surgery, neoadjuvant therapy, year of surgery and tumor size, involvement of other organs and tumor location on preoperative imaging) were included. 
Nearest neighbor matching was performed in a 1:1 ratio without replacement and a caliper width of 0.01 was specified. In order to be able to calculate propensity scores for all patients, missing baseline variables were imputed using single imputation based on predictive mean matching.

\section{$\underline{\text { Statistical analysis }}$}

Data were analyzed using IBM SPSS Statistics for Windows version 24.0 (IBM Corp., Armonk NY) and R Statistical Software version i386 3.3.3 (Foundation for Statistical Computing, Vienna, Austria). Analyses were performed according to the intention-to-treat principle. Normally distributed continuous data are presented as means with standard deviations (SDs) and were compared using the two independent samples t-test. Non-normally distributed continuous data are presented as medians with interquartile ranges (IQRs) and were compared using the Mann-Whitney $U$ test. Categorical data are presented as frequencies with percentages, and were compared using the Chisquare or Fisher's exact test, as appropriate. Survival curves were plotted according to the KaplanMeier method and comparison of survival probabilities was performed using the log rank (MantelCox) test and a Cox proportional hazards model. After matching, normally distributed continuous data were compared using the paired samples t-test. ${ }^{23}$ For non-normally distributed continuous data, the Wilcoxon signed rank test was used. Categorical data were compared using the McNemar's test. Comparison of survival probabilities after matching was performed using a stratified log-rank and a Cox proportional hazards model with shared frailty. ${ }^{24} \mathrm{~A}$ p-value below 0.05 was considered statistically significant. 


\section{Results}

\section{$\underline{\text { Participating centers }}$}

Participating centers performed a median of 93 [59 - 165] pancreatic resections per year, including, a median of 30 [20-59] distal pancreatectomies (all indications), 14 [6 - 25] distal pancreatectomies for PDAC and 15 MIDPs [10-26] per year.

\section{$\underline{\text { Total cohort }}$}

In total, 1297 patients were identified, of whom 85 were excluded for reasons shown in Figure 1, leaving 1212 patients for analysis. The total cohort consisted of 356 MIDPs (29\%) of which 16 (4\%) robot-assisted distal pancreatectomies, as shown in Table 1 (total cohort). Tumor involvement of other organs was less often seen on preoperative imaging in the MIDP group ( $6 \%$ vs. $13 \%, P=0.001$ ) and less neoadjuvant chemotherapy was used in the MIDP group ( $3 \%$ vs. $11 \%, P \leq 0.001)$. Intraoperative outcomes are presented in Table 2 (total cohort). Conversion from MIDP to ODP occurred in 65 patients (18\%). Postoperative length of hospital stay was shorter after MIDP (median 8 [ 5-12] vs. 9 [7-14] days, $P \leq 0.001$ ). All pathology outcomes are shown in Table 3 (total cohort). The median amount of retrieved lymph nodes was lower for MIDP compared with ODP (14 [8-22] vs. 18 [11-28] nodes, $P<0.001$ ) (Table 3, total cohort). The R0 resection rate was higher after MIDP compared with ODP ( $67 \%$ vs. $60 \%, P=0.015)$. All postoperative outcomes are shown in Table 4 total cohort. The overall survival curve stratified by procedure type is shown in supplementary figure 1 .

\section{Matched cohort}

Of all MIDPs, $96 \%$ could be matched successfully to an ODP control. As shown in Table 1 (matched cohort), significant differences in baseline characteristics were no longer present after matching. Table 2 (matched cohort) shows intra-operative outcomes. Conversion from MIDP to ODP occurred in 62 patients (19\%). Median blood loss was lower during MIDP compared with ODP (200 [60 - 400] vs. $300[150-500] \mathrm{mL}, P=0.001)$. Splenectomy (93\% vs. $97 \%, P=0.01)$, resection of Gerota's fascia 
( $31 \%$ vs. $60 \%$ patients, $P<0.001$ ) and vascular resections ( $6 \%$ vs. $11 \%, P=0.012$ ) were performed less frequently during MIDP compared with ODP. An adrenal gland resection was more often performed during MIDP compared with ODP ( $11 \%$ vs. $6 \%, P=0.029$ ). Table 3 (matched cohort) shows that the median lymph node retrieval was less during MIDP (14 [8-22] vs. 22 [14-31] nodes, $P<0.001)$ whereas the $\mathrm{RO}$ resection rate was higher in the MIDP group ( $67 \%$ vs. $58 \%, P=0.019)$. Lymphovascular invasion ( $56 \%$ vs. $71 \%$ patients, $P<0.001$ ) and perineural tumor invasion ( $63 \%$ vs. $75 \%$ patients, $p<0.001$ ) were less often seen in the MIDP group. No statistical significant differences in postoperative complications between MIDP and ODP were seen (Table 4, matched cohort). MIDP was associated with shorter postoperative hospital stay compared with ODP (8 [6-12] vs. 9 [7-14] days, $P<0.001)$. The median follow-up time was 13 (range: 0 - 84) months. Median overall survival was comparable for both procedures ( 28 [95\% Cl $22-34]$ vs. 31 [95\% Cl $26-36]$ months, $P=0.774)$ The Hazard Ratio, determined with a Cox proportional hazard regression with shared frailty analysis was $1.025(95 \% \mathrm{Cl}$ $0.75-1.27)$ for MIDP compared with ODP $(P=0.85)$. 


\section{Discussion}

This large pan-European retrospective propensity score matched cohort study on MIDP vs. ODP for PDAC confirms short term clinical advantages of MIDP, more specifically in terms of less intraoperative blood loss and shorter postoperative hospital stay. Overall survival was comparable after both procedures but the oncological safety of MIDP for PDAC, however, remains unclear as RO resection rate was higher but, Gerota's fascia was resected less often and lymph node retrieval was lower in MIDP. Matching did not influence these results, but, this does not exclude the presence of treatment allocation bias.

Three other matched cohorts specifically focusing on MIDP vs. ODP for patients with PDAC were published, one study in 102 patients used propensity score matching ${ }^{25}$ and two studies in 51 and 93 patients which used case matching. ${ }^{26,27}$ Reduced length of hospital stay after MIDP was reported in two studies ${ }^{25,27}$ and less intraoperative blood loss in one study ${ }^{27}$. As in the current study, none of the previously published studies reported a difference in postoperative complication rates.

The three previous matched cohorts did not report significant differences in R0 resection rates although the absolute risk difference between MIDP and ODP did favour MIDP in all cohorts and ranged from $8 \%$ to $9 \%$, which is similar to the $9 \%$ found in our study (Table 3, matched cohort). ${ }^{26,27}$ It should be noted that comparisons of $\mathrm{RO}$ resection rates in the literature have to be considered with caution, as RO rates are influenced by the definition used (no involvement of the margin or a distance between the margin and the tumor of at least $1 \mathrm{~mm}$ ) and method of margin assessment (transection margin alone or also circumferential margins) which may vary per pathologist and per institution. A systematic review illustrated this problem as it reported RO margin rates in large randomized controlled trials for resected PDAC as ranging from $17 \%$ to $100 \%{ }^{28}$ 
The number of retrieved lymph nodes also did not differ significantly in the previous matched cohorts, whereas in the current study we did find that a median of 8 less lymph nodes were retrieved during MIDP (Table 3, matched cohort). The amount of retrieved lymph nodes depends on the extend of the lymphadenectomy performed. The ISGPS definition of a standard lymphadenectomy ${ }^{29}$ recommends removal of lymph node station 10, 11 and 18 for body and tail tumors. Additional removal of station 9 is suggested in case of tumors confined to the area of the body of the pancreas. However, data on the type of lymphadenectomy performed was not available in this study, and since no evidence on the number of lymph nodes that should be resected is available the clinical relevance of our finding remains uncertain.

It is interesting to assess at details of surgical technique for instance, splenectomy, resection of Gerota's fascia and left adrenal gland resection, which are suggested to be relevant in achieving an RO resection and adequate lymphadenectomy. ${ }^{30-32}$ Both in the total and the matched cohort we found splenectomy and resection of Gerota's fascia to be less often performed in the MIDP group (Table 2). Adrenal gland resection on the other hand, was surprisingly performed more often in the MIDP group compared with ODP. It is unclear whether the differences found were related to the incapability to perform these steps minimally invasive or open or, whether surgeons did not consider these required for the cancers they resected, indicating that, despite matching, different tumors were present in the MIDP group.

The concerns on the oncological safety of MIPD for PDAC, could be related to worries about the ability to perform a RO resection or adequate lymphadenectomy. Standardized techniques have been described for MIDP in PDAC ${ }^{31}$, following the RAMPS technique as described by Strassberg ${ }^{30,32}$. MIDP for PDAC, should include standardized lymphadenectomy, resection of Gerota's fascia to reduce the risk of incomplete resection on the posterior margin as well as a 'no-touch approach', by lifting the pancreas using a hanging maneuver. This approach permits good views and access to the posterior aspect of the pancreas allowing for resection of Gerota's fascia and the adrenal gland, if needed. 
Therefore, with proper patient selection differences in RO resection rate, lymph node retrieval and, Gerota and adrenal gland resection should not be a concern in experienced hands.

No significant differences in overall survival have been reported for MIDP vs. ODP in PDAC ${ }^{25-27}$ and overall survival ranged from 14 to 16 months ${ }^{26,27}$. Although the current study neither found a significant difference in survival between groups, the reported survival was overall higher, ranging from 29 (MIDP) and 31 (ODP) months (Table 4, matched cohort). On the other hand, several large non-matched studies have reported survival times comparable to our study. ${ }^{25,33}$

Despite the clear strengths of this study, some limitations have to be discussed. First, most data were collected retrospectively which could have possibly led to underreporting of postoperative outcomes such as complications. Second, missing data were present. However, no differences between the baseline characteristics before and after imputation were present (Supplementary Table 1). For optimal transparency, all missing variables were reported and data should be interpreted in perspective to them. Third, despite our attempt to minimize the influence of treatment allocation bias, by applying PSM, treatment allocation bias may still have influenced outcomes in the matched cohort. Although we managed to correct for differences in baseline variables, the difference in lymphovascular and perineural tumor invasion between the MIDP and ODP group (Table 3, matched cohort) suggests that less aggressive tumors have been selected for the minimally invasive approach. The absence of these factors are associated with better survival in the literature, ${ }^{26,34}$ and as a consequence, this could influence the results. Lastly, the variation in techniques, reflected in the inclusion and sparing of the Gerota's fascia by different surgeons and in different centers, the lack of information on the location of lymph nodes resected represent a serious challenge to the comparison of homogenous groups

Due to the unknown clinical relevance of these findings in the present study, the oncological safety of MIDP remains uncertain. Standardization and agreement with regards to intraoperative 
techniques (splenectomy, lymphadenectomy, adrenal gland and Gerota's fascia resection) is required in order to be able to further investigate this subject. The E-MIPS group is currently preparing for the DIPLOMA-trial (Distal Pancreatectomy, Minimally Invasive or Open for PDAC) which will further investigate non-inferiority of MIDP vs. ODP for PDAC in a multicenter randomized setting. Such a study should include standardized surgical technique (e.g. regarding splenectomy, Gerota's fascia and lymphadenectomy) and standardized pathology assessment and reporting.

\section{Acknowledgements}

We acknowledge all E-MIPS participants for contributing to this study.

\section{Collaborators}

G Sergeant, department of surgery, Jessa Ziekenhuis, Hasselt, Belgium; S Berti, department of surgery, St. Andrea Hospital, La Spezia, Italy. 


\section{References}

1. Cuschieri A. Laparoscopic surgery of the pancreas. J R Coll Surg Edinb 1994; 39(3): 178-84.

2. Nigri GR, Rosman AS, Petrucciani N, et al. Metaanalysis of trials comparing minimally invasive and open distal pancreatectomies. Surg Endosc 2011; 25(5): 1642-51.

3. Jin $\mathrm{T}$, Altaf $\mathrm{K}$, Xiong JJ, et al. A systematic review and meta-analysis of studies comparing laparoscopic and open distal pancreatectomy. HPB (Oxford) 2012; 14(11): 711-24.

4. Jusoh AC, Ammori BJ. Laparoscopic versus open distal pancreatectomy: a systematic review of comparative studies. Surg Endosc 2012; 26(4): 904-13.

5. Pericleous S, Middleton N, McKay SC, Bowers KA, Hutchins RR. Systematic review and metaanalysis of case-matched studies comparing open and laparoscopic distal pancreatectomy: is it a safe procedure? Pancreas 2012; 41(7): 993-1000.

6. Sui CJ, Li B, Yang JM, Wang SJ, Zhou YM. Laparoscopic versus open distal pancreatectomy: a meta-analysis. Asian J Surg 2012; 35(1): 1-8.

7. Venkat R, Edil BH, Schulick RD, Lidor AO, Makary MA, Wolfgang CL. Laparoscopic distal pancreatectomy is associated with significantly less overall morbidity compared to the open technique: a systematic review and meta-analysis. Ann Surg 2012; 255(6): 1048-59.

8. Nakamura M, Nakashima H. Laparoscopic distal pancreatectomy and pancreatoduodenectomy: is it worthwhile? A meta-analysis of laparoscopic pancreatectomy. J Hepatobiliary Pancreat Sci 2013; 20(4): 421-8.

9. Drymousis $P$, Raptis DA, Spalding $D$, et al. Laparoscopic versus open pancreas resection for pancreatic neuroendocrine tumours: a systematic review and meta-analysis. HPB (Oxford) 2014; 16(5): 397-406.

10. Mehrabi A, Hafezi M, Arvin J, et al. A systematic review and meta-analysis of laparoscopic versus open distal pancreatectomy for benign and malignant lesions of the pancreas: it's time to randomize. Surgery 2015; 157(1): 45-55.

11. Abu Hilal M, Hamdan M, Di Fabio F, Pearce NW, Johnson CD. Laparoscopic versus open distal pancreatectomy: a clinical and cost-effectiveness study. Surg Endosc 2012; 26(6): 1670-4.

12. Riviere D, Gurusamy KS, Kooby DA, et al. Laparoscopic versus open distal pancreatectomy for pancreatic cancer. Cochrane Database Syst Rev 2016; 4: CD011391.

13. de Rooij T, Besselink MG, Shamali A, et al. Pan-European survey on the implementation of minimally invasive pancreatic surgery with emphasis on cancer. HPB (Oxford) 2016; 18(2): 170-6.

14. van Hilst J, de Rooij $\mathrm{T}$, Abu Hilal $\mathrm{M}$, et al. Worldwide survey on opinions and use of minimally invasive pancreatic resection. HPB (Oxford) 2017; 19(3): 190-204.

15. von Elm E, Altman DG, Egger M, Pocock SJ, Gøtzsche PC, Vandenbroucke JP. The Strengthening the Reporting of Observational Studies in Epidemiology (STROBE) statement: guidelines for reporting observational studies. The Lancet 2007; 370(9596): 1453-7.

16. Cancer TiAfRo. WHO Classification of Tumours of the Digestive System 4th edition. 2010.

17. Clavien PA, Barkun J, de Oliveira ML, et al. The Clavien-Dindo classification of surgical complications: five-year experience. Ann Surg 2009; 250(2): 187-96.

18. Bassi C, Dervenis C, Butturini G, et al. Postoperative pancreatic fistula: an international study group (ISGPF) definition. Surgery 2005; 138(1): 8-13.

19. Wente MN, Bassi C, Dervenis C, et al. Delayed gastric emptying (DGE) after pancreatic surgery: a suggested definition by the International Study Group of Pancreatic Surgery (ISGPS). Surgery 2007; 142(5): 761-8.

20. Wente MN, Veit JA, Bassi C, et al. Postpancreatectomy hemorrhage (PPH): an International Study Group of Pancreatic Surgery (ISGPS) definition. Surgery 2007; 142(1): 20-5.

21. Mangram AJ, Horan TC, Pearson ML, Silver LC, Jarvis WR. Guideline for Prevention of Surgical Site Infection, 1999. Centers for Disease Control and Prevention (CDC) Hospital Infection 
Control Practices Advisory Committee. Am J Infect Control 1999; 27(2): 97-132; quiz 3-4; discussion 96.

22. Pathologists. Standards and Minimum Datasets for Reporting Cancers Minimum dataset for the histopathological reporting of pancreatic, ampulla of Vater and bile duct carcinoma. London R Coll Pathol 2002.

23. Austin PC. Comparing paired vs non-paired statistical methods of analyses when making inferences about absolute risk reductions in propensity-score matched samples. Stat Med 2011; 30(11): 1292-301.

24. Austin PC. The use of propensity score methods with survival or time-to-event outcomes: reporting measures of effect similar to those used in randomized experiments. Stat Med 2014; 33(7): 1242-58.

25. Shin SH, Kim SC, Song KB, et al. A comparative study of laparoscopic vs. open distal pancreatectomy for left-sided ductal adenocarcinoma: a propensity score-matched analysis. $J$ Am Coll Surg 2015; 220(2): 177-85.

26. Kooby DA, Hawkins WG, Schmidt CM, et al. A multicenter analysis of distal pancreatectomy for adenocarcinoma: is laparoscopic resection appropriate? J Am Coll Surg 2010; 210(5): 77985, 86-7.

27. Zhang $M$, Fang $R$, Mou $Y$, et al. LDP vs ODP for pancreatic adenocarcinoma: a case matched study from a single-institution. BMC Gastroenterol 2015; 15(1): 182.

28. Butturini G, Stocken DD, Wente MN, et al. Influence of resection margins and treatment on survival in patients with pancreatic cancer: meta-analysis of randomized controlled trials. Arch Surg 2008; 143(1): 75-83; discussion

29. Tol JA, Gouma DJ, Bassi C, et al. Definition of a standard lymphadenectomy in surgery for pancreatic ductal adenocarcinoma: a consensus statement by the International Study Group on Pancreatic Surgery (ISGPS). Surgery 2014; 156(3): 591-600.

30. Strasberg SM, Linehan DC, Hawkins WG. Radical antegrade modular pancreatosplenectomy procedure for adenocarcinoma of the body and tail of the pancreas: ability to obtain negative tangential margins. J Am Coll Surg 2007; 204(2): 244-9.

31. Abu Hilal M, Richardson JR, de Rooij T, Dimovska E, Al-Saati H, Besselink MG. Laparoscopic radical 'no-touch' left pancreatosplenectomy for pancreatic ductal adenocarcinoma: technique and results. Surg Endosc 2016; 30(9): 3830-8.

32. Strasberg SM, Drebin JA, Linehan D. Radical antegrade modular pancreatosplenectomy. Surgery 2003; 133(5): 521-7.

33. Sulpice L, Farges O, Goutte N, et al. Laparoscopic Distal Pancreatectomy for Pancreatic Ductal Adenocarcinoma: Time for a Randomized Controlled Trial? Results of an All-inclusive National Observational Study. Ann Surg 2015; 262(5): 868-74.

34. Schorn S, Demir IE, Haller B, et al. The influence of neural invasion on survival and tumor recurrence in pancreatic ductal adenocarcinoma - A systematic review and meta-analysis. Surg Oncol 2017; 26(1): 105-15. 


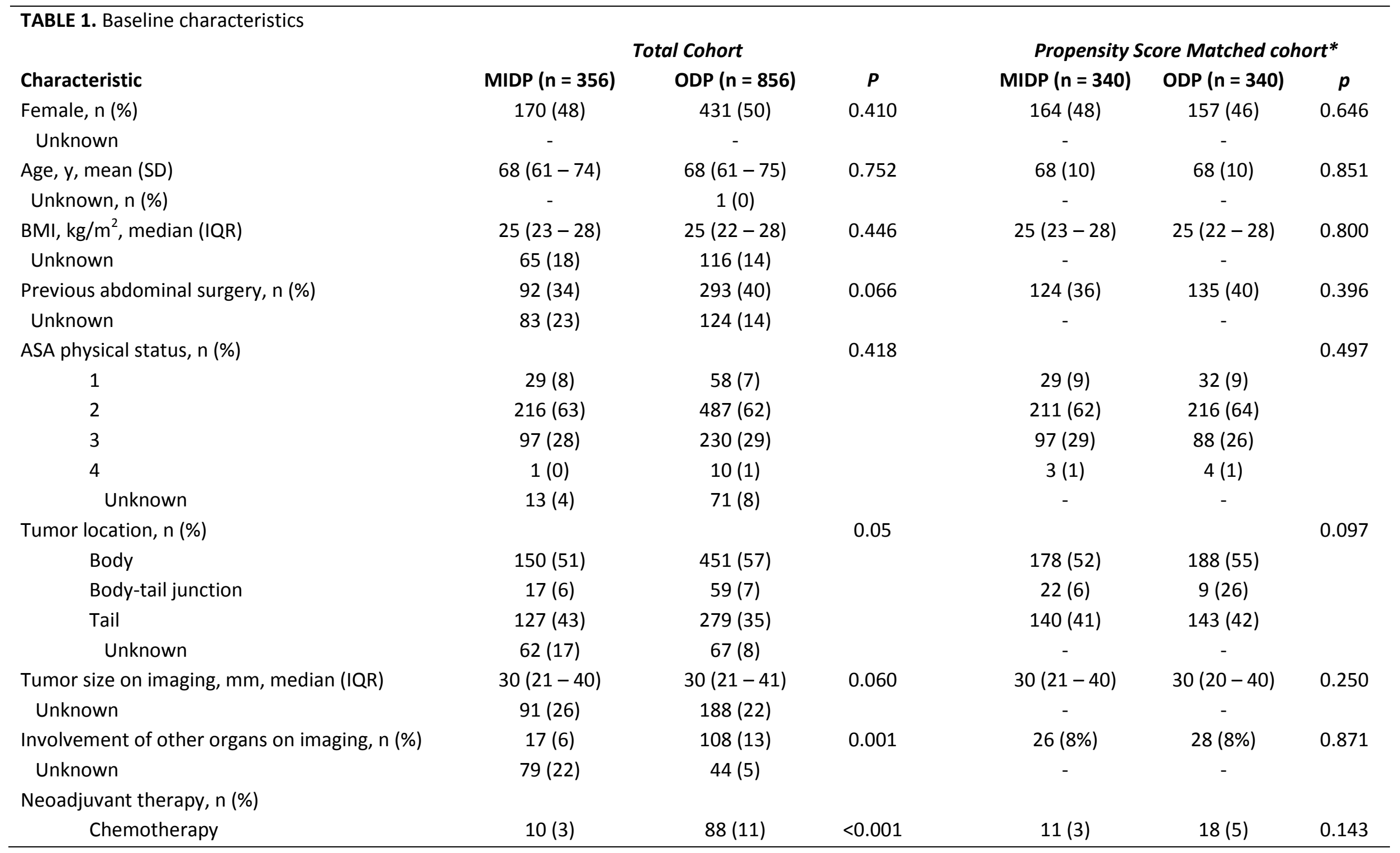




\begin{tabular}{|c|c|c|c|c|c|c|}
\hline Unknown & $5(1)$ & $19(2)$ & & - & - & \\
\hline Radiotherapy & $4(0)$ & $16(2)$ & 0.352 & $4(1)$ & 7 (2) & 0.549 \\
\hline Unknown & $7(2)$ & $18(2)$ & & - & - & \\
\hline
\end{tabular}

*In the matched cohort we have no unknown baseline data due to imputation as described in the methods section. 


\begin{tabular}{|c|c|c|c|c|c|}
\hline \multicolumn{3}{|c|}{ Total Cohort } & \multicolumn{3}{|c|}{ Propensity Score Matched cohort* } \\
\hline $16(4)$ & - & & $16(5)$ & - & - \\
\hline $65(18)$ & - & - & $62(19)$ & - & - \\
\hline- & - & & - & & \\
\hline $17(26)$ & - & & $17(27)$ & & \\
\hline $17(26)$ & - & & $16(26)$ & & \\
\hline $4(6)$ & - & & $4(6)$ & & \\
\hline $3(5)$ & - & & $3(5)$ & & \\
\hline $1(2)$ & - & & $1(2)$ & & \\
\hline $8(12)$ & - & & $8(13)$ & & \\
\hline $239(180-290)$ & $240(182-297)$ & 0.520 & $240(180-295)$ & $230(178-286)$ & 0.626 \\
\hline - & $1(0)$ & & - & - & \\
\hline $77(30)$ & $289(41)$ & 0.002 & $66(31)$ & $129(60)$ & $<0.001$ \\
\hline $96(27)$ & $146(17)$ & & $124(36)$ & $124(36)$ & \\
\hline $33(11)$ & $65(8)$ & 0.165 & $29(11)$ & $15(6)$ & 0.029 \\
\hline $51(14)$ & $31(4)$ & & $71(21)$ & $71(21)$ & \\
\hline $41(12)$ & $133(16)$ & 0.120 & $33(11)$ & $35(12)$ & 0.901 \\
\hline $27(8)$ & $29(3)$ & & $52(15)$ & $52(15)$ & \\
\hline 4 & 16 & & 3 & 1 & \\
\hline 6 & 14 & & 5 & 5 & \\
\hline
\end{tabular}




\begin{tabular}{|c|c|c|c|c|c|c|}
\hline Colectomy (partial) & 14 & 44 & & 10 & 14 & \\
\hline Small bowel (partial) & 7 & 21 & & 6 & 3 & \\
\hline Gastrectomy (partial) & 10 & 63 & & 10 & 17 & \\
\hline Unknown & 2 & 2 & & 2 & 1 & \\
\hline Vascular resection***, n (\%) & $19(5)$ & $92(11)$ & 0.003 & $19(6)$ & $38(11)$ & 0.012 \\
\hline Unknown & - & - & & - & - & \\
\hline Postomesenteric vein & $12(63)$ & $78(85)$ & & $12(53)$ & $34(68)$ & \\
\hline
\end{tabular}

DP indicates distal pancreatectomy; IQR, interquartile range; MIDP, minimally invasive distal pancreatectomy; ODP, open distal pancreatectomy.

*Due to the use of paired tests, analyses could only be performed on data of complete pairs.

**Procedure with additional organ resection besides DP, splenectomy or adrenalectomy. In some procedures multiple organ resections were performed.

***Procedure with additional vascular resection besides splenic vessels. 


\begin{tabular}{|c|c|c|c|c|c|c|}
\hline \multirow[b]{2}{*}{ Characteristic } & \multicolumn{3}{|c|}{ Total Cohort } & \multicolumn{3}{|c|}{ Propensity Score Matched cohort* } \\
\hline & MIDP (n = 356) & ODP $(n=856)$ & $P$ & MIDP (n = 340) & ODP $(n=340)$ & $p$ \\
\hline Tumor size, mm, median (IQR) & $34(25-45)$ & $34(23-47)$ & 0.690 & $35(25-45)$ & $30(23-45)$ & 0.970 \\
\hline Unknown & $10(3)$ & $41(5)$ & & $23(7)$ & $23(7)$ & \\
\hline Tumor stage & & & 0.100 & & & 0.917 \\
\hline $\mathrm{T} 1$ & $24(7)$ & $75(9)$ & & $22(7)$ & $27(8)$ & \\
\hline $\mathrm{T} 2$ & $58(17)$ & $100(12)$ & & $54(16)$ & $46(14)$ & \\
\hline T3 & $257(74)$ & $597(74)$ & & $242(74)$ & $239(73)$ & \\
\hline $\mathrm{T} 4$ & $10(3)$ & $37(5)$ & & $10(3)$ & $16(5)$ & \\
\hline Unknown & $7(2)$ & $47(5)$ & & $12(4)$ & $12(4)$ & \\
\hline Lymph node stage & & & 0.012 & & & 0.007 \\
\hline NO & $153(44)$ & $296(36)$ & & $147(44)$ & $112(34)$ & \\
\hline N1 & $198(56)$ & $530(64)$ & & $184(56)$ & $219(66)$ & \\
\hline Unknown & $5(1)$ & $30(4)$ & & $9(3)$ & $9(3)$ & \\
\hline Lymph nodes retrieved, median (IQR) & $14(8-22)$ & $18(11-28)$ & $<0.001$ & $14(8-22)$ & $22(14-31)$ & $<0.001$ \\
\hline Unknown & $11(3)$ & $10(1)$ & & $15(4)$ & $15(4)$ & \\
\hline Tumor positive lymph nodes retrieved, median (IQR) & $1(0-2)$ & $1(0-3)$ & $<0.001$ & $1(0-2)$ & $2(0-4)$ & $<0.001$ \\
\hline Unknown & $5(1)$ & $21(2)$ & & $9(3)$ & $9(3)$ & \\
\hline $\mathrm{RO}$ resection**, n (\%) & $235(67)$ & $501(60)$ & 0.015 & $218(67)$ & $188(58)$ & 0.019 \\
\hline Unknown & $7(2)$ & $18(2)$ & & $14(4)$ & $14(4)$ & \\
\hline Lymphovascular invasion, n (\%) & $183(56)$ & $508(65)$ & 0.002 & $164(56)$ & $210(71)$ & $<0.001$ \\
\hline Unknown & $28(8)$ & $80(9)$ & & $46(14)$ & $46(14)$ & \\
\hline Perineural invasion, n (\%) & $236(72)$ & $648(82)$ & $<0.001$ & $214(63)$ & $255(75)$ & $<0.001$ \\
\hline Unknown & $28(8)$ & $62(7)$ & & $43(13)$ & $43(13)$ & \\
\hline
\end{tabular}




\begin{tabular}{|c|c|c|c|c|c|c|}
\hline \multirow[b]{2}{*}{ Characteristic } & \multicolumn{3}{|c|}{ Complete Cohort } & \multicolumn{3}{|c|}{ Propensity Score Matched cohort } \\
\hline & MIDP $(n=356)$ & ODP $(n=856)$ & $\boldsymbol{P}$ & MIDP $(n=340)$ & ODP $(n=340)$ & $p$ \\
\hline Clavien-Dindo score $\geq$ III complications, n (\%) & $62(17)$ & $186(22)$ & 0.088 & $61(18)$ & $70(21)$ & 0.431 \\
\hline Unknown & $0(0)$ & $1(0)$ & & $0(0)$ & $0(0)$ & \\
\hline POPF grade B/C*, n (\%) & $67(19)$ & $163(19)$ & 0.931 & $65(19)$ & $67(20)$ & 0.921 \\
\hline Unknown & $1(0)$ & $2(0)$ & & $1(0)$ & $1(0)$ & \\
\hline DGE grade $\mathrm{B} / \mathrm{C}^{* *}, \mathrm{n}(\%)$ & $8(2)$ & $62(7)$ & 0.002 & $8(3)$ & $17(5)$ & 0.108 \\
\hline Unknown & $33(9)$ & $18(2)$ & & $30(9)$ & $30(9)$ & \\
\hline PPH grade $B / C^{* *}, \mathrm{n}(\%)$ & $15(5)$ & $29(3)$ & 0.365 & $15(5)$ & $16(5)$ & $>0.999$ \\
\hline Unknown & $29(8)$ & $18(2)$ & & $26(8)$ & $26(8)$ & \\
\hline Surgical site infection, n (\%) & $4(1)$ & $34(4)$ & 0.022 & $4(1)$ & $9(3)$ & 0.267 \\
\hline Unknown & $50(14)$ & $18(2)$ & & $46(14)$ & $46(14)$ & \\
\hline Length of hospital stay, $d$, median (IQR) & $8(5-12)$ & $9(7-14)$ & $<0.001$ & $8(6-12)$ & $9(7-14)$ & $<0.001$ \\
\hline Unknown & $3(1)$ & $13(2)$ & & $7(2)$ & $7(2)$ & \\
\hline Readmission, $\mathrm{n}(\%)$ & $41(13)$ & $113(14)$ & 0.580 & $38(13)$ & $41(14)$ & 0.804 \\
\hline Unknown & $36(10)$ & $53(6)$ & & $44(13)$ & $44(13)$ & \\
\hline 90-day mortality, n (\%) & $8(2)$ & $28(4)$ & 0.256 & $7(2)$ & $8(3)$ & $>0.999$ \\
\hline Unknown & $7(2)$ & $73(9)$ & & $41(12)$ & $41(12)$ & \\
\hline Adjuvant chemotherapy, n (\%) & $226(74)$ & $482(73)$ & 0.700 & $165(76)$ & $159(73)$ & 0.561 \\
\hline Unknown & $51(14)$ & $195(23)$ & & $122(36)$ & $122(36)$ & \\
\hline Time until start adjuvant chemotherapy, $d$, median (IQR) & $54(41-69)$ & $57(43-71)$ & & $54(41-67)$ & $57(45-69)$ & 0.778 \\
\hline Unknown & $118(52)$ & $262(54)$ & & $315(93)$ & $315(93)$ & \\
\hline
\end{tabular}

IQR, interquartile range; MIDP, minimally invasive distal pancreatectomy; ODP, open distal pancreatectomy.

*According to the International Study Group on Pancreatic Fistula definition

** According to the International Study Group on Pancreatic Surgery definition 
Figure 1: Flow diagram

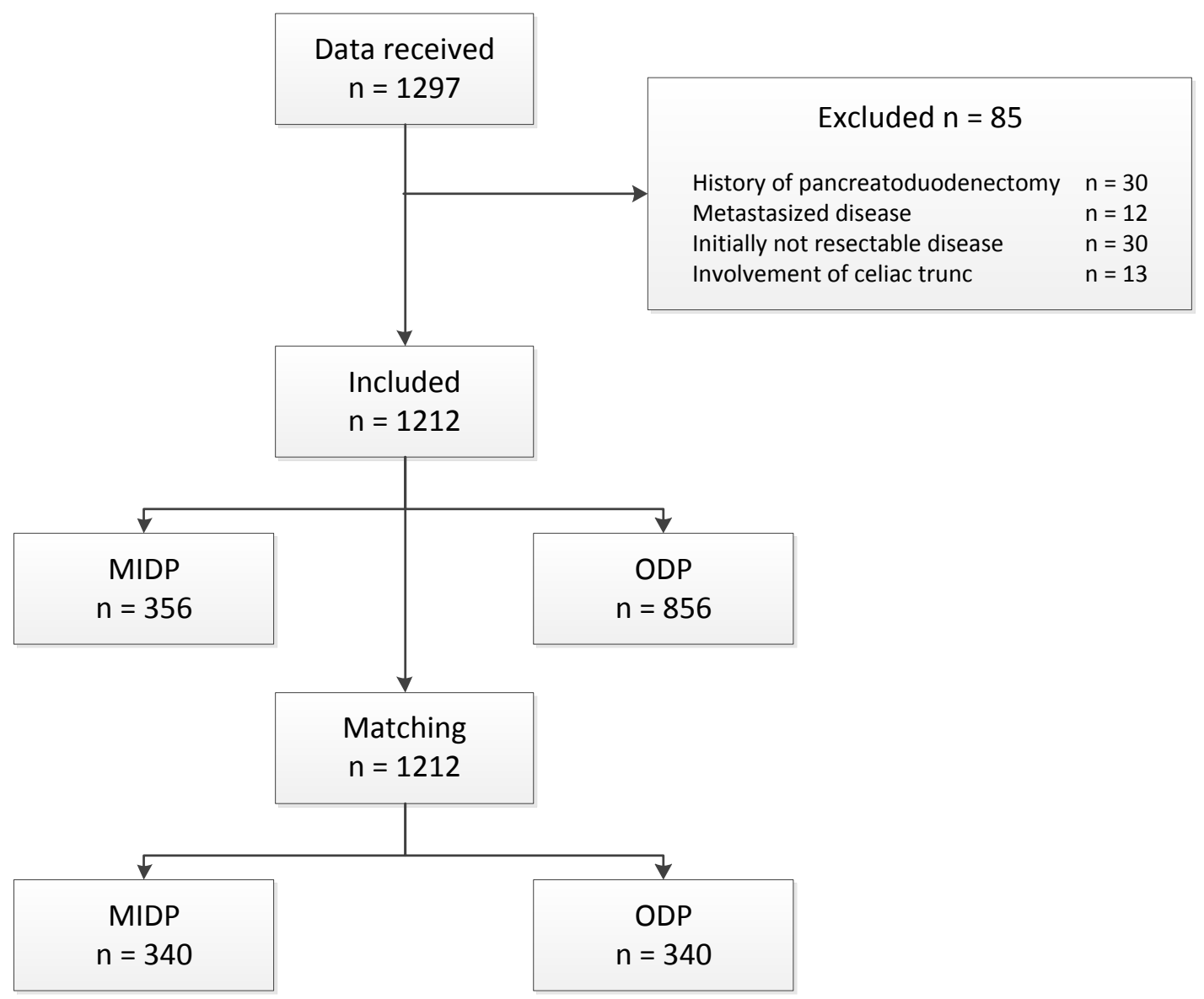


Figure 2: Kaplan-Meier overall survival matched cohort. Stratified Log-Rank test, $P=0.774$. Cox proportional hazards model with shared frailty, $P=0.85$

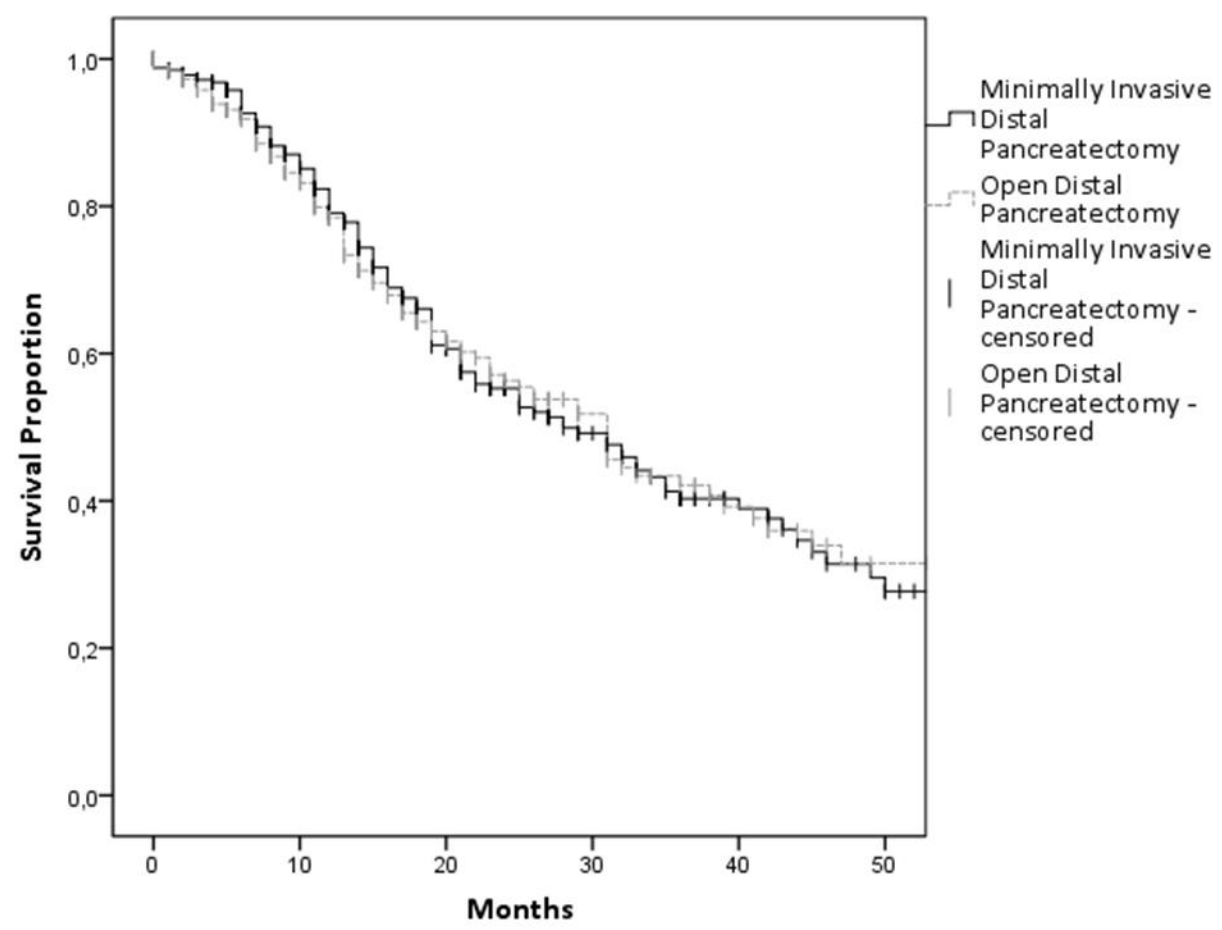

\begin{tabular}{|c|c|c|c|c|c|c|}
\hline \multicolumn{3}{|c|}{ Number at risk } & \multirow[b]{2}{*}{118} & \multirow[b]{2}{*}{64} & \multirow[b]{2}{*}{30} & \multirow[b]{2}{*}{16} \\
\hline MIDP & 340 & 224 & & & & \\
\hline ODP & 340 & 184 & 92 & 50 & 25 & 12 \\
\hline
\end{tabular}




\begin{tabular}{|c|c|c|c|c|c|c|}
\hline \multicolumn{7}{|c|}{ Supplementary table 1. Baseline characteristics after imputation } \\
\hline Female, n (\%) & $170(48)$ & $431(50)$ & 0.410 & $170(48)$ & $431(50)$ & 0.410 \\
\hline Age, $y$, mean (SD) & $68(61-74)$ & $68(61-75)$ & 0.752 & $67(10)$ & $67(10)$ & 0.772 \\
\hline Unknown, n (\%) & - & $1(0)$ & & - & - & \\
\hline $\mathrm{BMI}, \mathrm{kg} / \mathrm{m}^{2}$, median (IQR) & $25(23-28)$ & $25(22-28)$ & 0.446 & $25(22-28)$ & $25(22-28)$ & 0.400 \\
\hline Unknown & $83(23)$ & $124(14)$ & & - & - & \\
\hline ASA physical status, $\mathrm{n}(\%)$ & & & 0.418 & & & 0.124 \\
\hline 1 & $29(8)$ & $58(7)$ & & $30(8)$ & $62(7)$ & \\
\hline 2 & $216(63)$ & $487(62)$ & & $226(64)$ & $520(61)$ & \\
\hline 3 & $97(28)$ & $230(29)$ & & $99(28)$ & $258(30)$ & \\
\hline Body-tail junction & $17(6)$ & $59(7)$ & & $22(6)$ & $61(7)$ & \\
\hline Tail & $127(43)$ & $279(35)$ & & $151(42)$ & $309(36)$ & \\
\hline Unknown & $62(17)$ & $67(8)$ & & - & - & \\
\hline Tumor size on imaging, mm, median (IQR) & $30(21-40)$ & $30(21-41)$ & 0.060 & $30(20-40)$ & $30(21-40)$ & 0.054 \\
\hline Unknown & $91(26)$ & $188(22)$ & & - & - & \\
\hline Involvement of other organs on imaging, $\mathrm{n}(\%)$ & $17(6)$ & $108(13)$ & 0.001 & $28(10 \%)$ & $120(15 \%)$ & 0.050 \\
\hline Unknown & $79(22)$ & $44(5)$ & & - & - & \\
\hline \multicolumn{7}{|l|}{ Neoadjuvant therapy, n (\%) } \\
\hline Chemotherapy & $10(3)$ & $88(11)$ & $<0.001$ & $10(3)$ & $88(11)$ & $<0.001$ \\
\hline
\end{tabular}




\begin{tabular}{lllll} 
Unknown & $5(1)$ & $19(2)$ & & - \\
Radiotherapy & $4(0)$ & $16(2)$ & 0.352 & $4(1)$ \\
Unknown & $7(2)$ & $18(2)$ & - \\
\hline
\end{tabular}

ASA indicates American Society of Anesthesiologists; BMI, body mass index; IQR, interquartile range; MIDP, minimally invasive distal pancreatectomy; ODP, open distal pancreatectomy; SD, standard deviation. 
Supplementary figure 1: Kaplan-Meier overall survival total cohort. Log-Rank test, $P=0.371$.

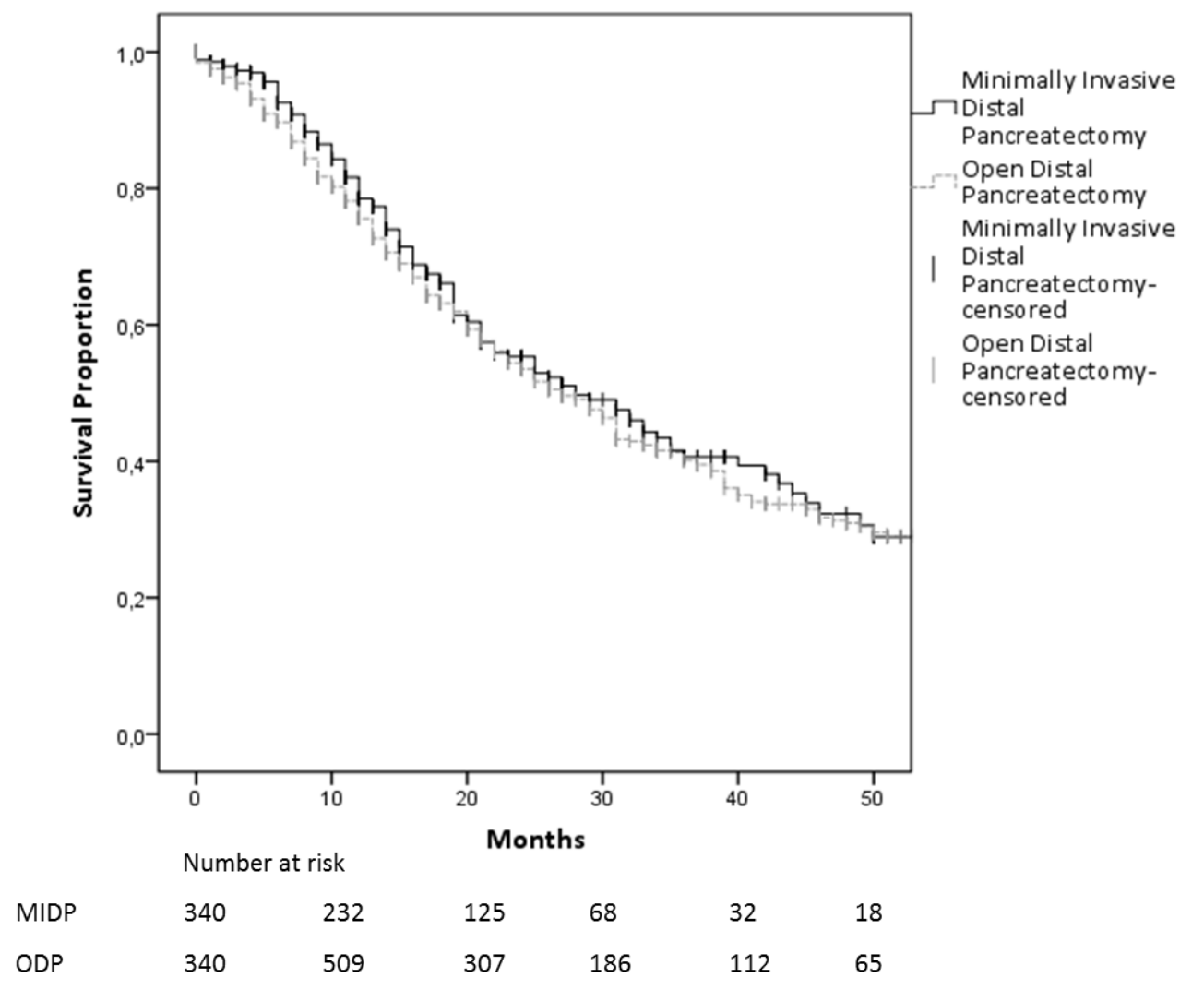

